\title{
Análisis Inicial de la Enseñanza del Idioma Español por Medio de Actividades Deportivas en Varsovia (Polonia)
}

\author{
Anna MALISZEWSKA
}

Datos de contacto:

Anna Maliszewska

Trilingual nursery, pre-school and primary/Crèche, maternelle et primaire trilingues anna.maliszewska@3languages. pl

\section{RESUMEN}

El presente artículo muestra la metodología utilizada para la enseñanza del idioma español por medio de las actividades deportivas (Educación Física) en la escuela trilingüe de Varsovia (Polonia). En este estudio han participado 102 niños y niñas de 7 a 12 años cuyo profesorado de actividades deportivas es nativo español y desarrolla la materia únicamente en dicho idioma. Los resultados muestran que en un periodo de entre 3 y 5 años, el alumnado adquiere un nivel de español igual o superior al B1. Pese a que no se ha podido establecer relación entre el aprendizaje de actividades deportivas y el nivel lingüístico, atendiendo a los resultados académicos de quienes tienen un nivel bajo de español, el contexto de las actividades deportivas se vislumbra como favorable en el desarrollo de la competencia motriz.

PALABRAS CLAVE: Actividad Física; Educación Física; Plurilingüismo; Interdisciplinaridad. 


\title{
Initial Analysis of Spanish Language Teaching through Sports Activities in Warsaw (Poland)
}

\begin{abstract}
This article shows the methodology used for teaching the Spanish language through sports activities (Physical Education) in the trilingual school in Warsaw (Poland). 102 boys and girls from 7 to 12 years old whose sport teachers are native Spanish speakers and teach the subject in their native langaueg have participated in this study. The results show that in a period of between 3 and 5 years, students acquire a level of Spanish equal to or greater than B1. Despite the fact that it has not been possible to establish a relationship between the learning of sports activities and the language level, considering the academic results of those who have a low level of Spanish, the context of sports activities is seen as favorable in the development of gross motor skills.
\end{abstract}

KEYWORDS: Physical Activity; Physical Education; Multilingualism; Interdisciplinary.

\section{Introducción}

Hoy en día la relación positiva entre el movimiento y los procesos cognitivos es un hecho aceptado. Jean Piaget fue el primer investigador que abordo la relación entre el ámbito cognitivo y el psicomotor centrando su estudo en el desarrollo intelectual de niños y niñas. Según su trabajo, los infantes reciben e interiorizan la información que procede principalmente del movimiento (Rodríguez y Vega, 2004; Woodburn, 1985). Posteriormente, han sido muchos los autores y autoras que han seguido esta linea de investigación, así, desde diferentes ámbitos se ha demostrado que la motricidad y el desarrollo cognitivo mantienen una destacada relación (Barajas, 2013) que favorece el conocimiento del entorno y del propio protagonista. Como apunta Bolaños (1991), los niños y niñas, a través del movimiento pueden lograr aprender a mejorar su rendimiento en todos los ámbitos de su vida.

Desde esta perspectiva, la Educación Física juega un papel fundamental en los procesos de aprendizaje de las personas, pero es si cabe, aún más importante en los primeros años de escolarización.

Pese a que el modo en el que se enseña y se aprende ha cambiado radicalmente desde los primeros estudios de Piaget, la relevancia del movimiento en los procesos cognitivos se presenta como una linea de investigación emergente. En un reciente estudio (Reloba, Chirosa, y Reigal, 2016) en el que se realizó una revisión sistemática de un total de 27 
artículos que analizaban los efectos del ejercicio físico sobre los procesos cognitivos de niños y niñas de 6 a 12 años, concluyó que la actividad física tiene una influencia positiva sobre los procesos cognitivos del alumnado si bien estos no parecen ser apreciables en su rendimiento académico a corto plazo.

La educación física viene siendo una disciplina que por sus características estructurales es elegida como área adecuada para el desarrollo conjunto de otras materias. En diferentes ámbitos se hace alusión a la interdisciplinariedad. La interdisciplinariedad es un modelo de generación de conocimiento en el que se ofrece una forma para superar la fragmentación del saber, no como una "reducción a la identidad" sino como toma de conciencia de la complejidad de las realidades que nos rodean (Grisolia, 2016). Así pues, existen estudios que vinculan la bondad de este tipo de maridaje principalmente con las matemáticas y el aprendizaje de las lenguas.

Vigotsky, defiende que el aprendizaje humano ocurre a través de la interacción social en un ambiente natural (Genesee 1999). Asimismo, encontramos autores que defienden que los idiomas se utilizan como elementos de transmisión y adquisición de contenidos linguïsticos y académicos en situaciones reales que giran alrededor de la comunicación y el significado (Cummins, 2002; García, 2005; Ramos y Ruíz Omenaca, 2011).

El estudio realizado por Ramos y Ruíz Omenaca (2011) describe una propuesta metodológica para la enseñanza del inglés a través de la Educación Física en programas bilingües español-inglés, y explica la importancia del Aprendizaje Integrado de Contenidos y Lenguas Extranjeras (AICLE), -en inglés, Content and Language Integrated Learning (CLIL)-, especificando la idoneidad de la Educación Física para la integración del lenguaje y contenidos en inglés.

El interés por el plurilingüismo se deriva de los cambios sociales y culturales que se abordan en la actualidad. La libre circulación junto con la globalización requiere que las nuevas generaciones sean capaces de comunicarse en diferentes idiomas. El Marco Común Europeo para las lenguas, surge con el objetivo de facilitar la labor a todos los agentes implicados en el proceso de enseñanza y aprendizaje de las lenguas, posibilitar una actuación más eficaz y contrastar los resultados obtenidos.

Siguiendo los dictados del Marco Común Europeo, se han estudiado diferentes metodologías que abordan los procesos de enseñanza aprendizaje de las lenguas. Uno de los más destacables es el ya mencionado Aprendizaje Integrado de Contenidos y Lenguas Extranjeras (AICLE).

El término AICLE fue creado en 1994 por David Marsh 163para definir una metodología que defiende que en los contextos escolares es mayor el éxito que se obtiene en el aprendizaje de las lenguas extranjeras cuando se imparten las materias comunes del curriculum, como las ciencias sociales 
o la educación física, en el idioma elegido en lugar de hacerlo de una manera aislada buscando situaciones que de algún modo se acerque al contexto de la lengua, pero siendo en cualquier caso situaciones expresamente creadas y descontextualizadas. Es frecuente que se utilice como sinónimo de la inmersión lingüística, pero no es lo mismo. AICLE a menudo se utiliza como un término "paraguas" que recoge múltiples métodos de utilización del idioma como medio de instrucción.

Coyle (2002), plantea cuatro principios rectores sobre los cuales se puede construir un programa AICLE.

1. Contenido. Hace referencia al tema a desarrollar. Es el eje del proceso de aprendizaje y a partir de este se establece la adquisición de conocimientos, habilidades y competencias.

2. Comunicación. El lenguaje se presenta como el elemento que vehiculiza la comunicación y el aprendizaje. Han de aprender el lenguaje para comunicarse y utilizar el lenguaje para expresar su conocimiento. La implicación de los y las estudiantes tiene lugar de un modo diferente al que se da en el aprendizaje "clasico" de las lenguas. Va más allá del sistema gramatical.

3. Cognición. La eficacia de AICLE radica en el desafio que supone para el alumnado. Se aleja de los modelos de transferencia. AICLE consiste en facilitar que los aprendices "construyan" la comprensión superando desafios que se presentan en el proceso natural, independientemente de su edad o habilidad.

4. Cultura. La globalización trae consigo la apertura a diferentes cultural y lenguas. Estudiar a través de un idioma extranjero es fundamental para fomentar la comprensión internacional.

AICLE ha sido también un método muy alabado por La Comisión Europea (Commission Of the European Communities, 2003) porque es una metodología que puede proporcionar oportunidades efectivas para que el alumnado use sus nuevas habilidades lingüisticas en el momento en el que las está aprendiendo, en lugar de aprenderlas para su uso posterior. Es un método que "abre las puertas" a los idiomas para estudiantes con diferentes competencias fomentando la autoconfianza en estudiantes jóvenes y/o aquellos y aquellas que no han respondido bien a los modelos tradicionales de enseñanza de los idiomas. La característica que define al AICLE es la exposición al idioma sin requerir tiempo adicional en el plan de estudios. Por esta razón, la Comisión Europea decidió promocionar al profesorado para "Mejorar las competencias lingüísticas en general, y para promover la enseñanza de asignaturas no lingüísticas en lenguas extranjeras" (Resolucuón de la Comisión de la Unión Europea del 21 de noviembre de 2008).

La investigación en este campo (Coyle, Hood, y Marsh, 2010) ha demostrado también que el multilingüismo en la escuela, aprovechando los recursos, estrategias y destrezas que desarrolla el alumnado cuando aprende una lengua o materia, a través de los currículos integrados de las 
lenguas y áreas no lingüísticas, contribuye de manera eficaz al desarrollo de una competencia comunicativa en lenguas que optimiza y facilita los aprendizajes posteriores. En este sentido, el lenguaje no es el foco principal de atención, pero se usa como un medio de comunicación. Esto implica un aprendizaje significativo del idioma que se considera la base para el desarrollo de la competencia comunicativa, el objetivo final del aprendizaje de una lengua extranjera.

Vollmer (2006, p.7) añade los siguientes principios para la integración curricular de lenguas y contenidos en la escuela:

- El lenguaje es más que destrezas comunicativas.

- El lenguaje actúa en los procesos mentales y se usa en la ejecución de estos.

- El lenguaje es una herramienta para conceptualizar y vincular información.

- El lenguaje soporta actividades mentales y precisión en los procesos cognitivos especialmente válidos para la escritura académica.

- El lenguaje interviene en la resolución de tareas cognitivamente complejas carentes de contextualización.

- El lenguaje ayuda a estructurar el discurso y realizar funciones discursivas.

Asimismo, Vollmer (2006) afirma que el Aprendizaje Integrado de Lenguas Extranjeras y otros Contenidos Curriculares implica estudiar asignaturas como historia o ciencias naturales en una lengua distinta de la propia. AICLE resulta muy beneficioso tanto para el aprendizaje de lenguas extranjeras como para las asignaturas impartidas en dichas lenguas. El énfasis de AICLE en la "resolución de problemas" y "saber hacer cosas", hace que los y las estudiantes se sientan motivadas al poder resolver problemas y hacer cosas incluso en otras lenguas (Navés y Muñoz, 2000, en Gómez Muñiz, 2013)

Dakowska (1995; 2011) se concentra en las posibilidades de utilizar el método AICLE para adquirir la competencia comunicativa intercultural. Ella argumenta que la educación bilingüe es un método de enseñanza eficaz y de recursos para adquirir los objetivos de aprendizaje del idioma como objetivo y como contenido. También afirma que, al enseñar otras materias a través de una lengua diferente a la materna, el alumnado aumenta la competencia intercultural, indispensable para vivir en otra cultura. McKay (2012) sostiene que la cultura influye en la enseñanza de idiomas de dos maneras: lingüística y pedagógica. Lingüísticamente, afecta los niveles semántico, pragmático y discursivo del lenguaje; pedagógicamente, influye en la elección de los materiales del idioma porque el contenido cultural de los materiales del idioma y la base cultural de la 
metodología de enseñanza deben tenerse en cuenta al decidir sobre los materiales del idioma. 166

Existen diferentes métodos que acompañan a la metodologia AICLE. Uno de los más eficaces es el método OPOL (one parent-one lenguage). Este método fue el primer sistema que se reconoció para el desarrollo de habilidades bilingües en bebés. Su origen viene de las familias con miembros con distinta lengua materna, y surgió de una forma natural. Según Barron-Hauwaert (2004) fue Maurice Grammont en 1902 el primero en observar los resultados que se obtenían cuando cada uno de los padres hablaba al bebé en su propia lengua. Su conclusión fue que de esta forma se conseguía un desarrollo cerebral del niño/a perfecto llegando a dominar totalmente los dos idiomas antes de los 5 años de edad.

La globalización en la que está sumida la vida del S.XXI nos sitúa ante una sociedad necesariamente interconectada que requiere desarrollar competencias asociadas a la comunicación. Fruto de esta necesidad surgen los centros de enseñanza multilingües. El objetivo de este estudio es realizar un primer análisis sobre el posible efecto positivo del aprendizaje del español a través del modelo AICLE, dando a conocer los resultados de una experiencia que aborda el aprendizaje de este idioma a través de la asignatura Educacion Física (actividades deportivas) en una escuela trilingüe en Polonia.

\section{Método}

\section{Diseño y Metodología}

Se trata de un estudio de corte cuantitativo.

Las variables estudiadas son: sexo, grado en el que se encuentra el alumnado (curso), lengua maternal, nota en las actividades deportivas (EF), nota en español, así como los años que lleban estudiando español y el nivel lingüístico en el que se encuentran actualmente.

\section{Participantes}

Los participantes son 102 estudiantes de los cuales 48 son niños y 53 son niñas, todos y todas alumnos de $1^{\circ}$ a $6^{\circ}$ grado de educación primaria (de 7 a 12 años) de la International Trilingual School of Warsaw (Polonia).

\section{Procedimiento}

Tras obtener los permisos pertinentes se procedio al desarrollo del estudio. Durante todo el proceso, además de tener en cuenta las características personales del alumnado, principalmente las relacionadas con su/sus idioma/s de origen, se hizo el seguimiento del aprendizaje atendiendo a su evolución tanto a nivel físico, como a nivel lingüístico.

Para entender las características del estudio se considera interesante conocer el contexto así como la metodología de enseñanza-aprendizaje. 


\section{El Contexto}

Este estudio se sitúa en la escuela Internacional Trilingüe de Varsovia. Se trata de un centro privado en el que el nivel adquisitivo medio de las familias es alto.

Dicha escuela enseña en cinco idiomas. En cada grado se enseña obligatoriamente en inglés y polaco. A la edad de un año los padres de los y las alumnas deciden sobre la selección del tercer idioma. Escogen entre español, francés y chino.

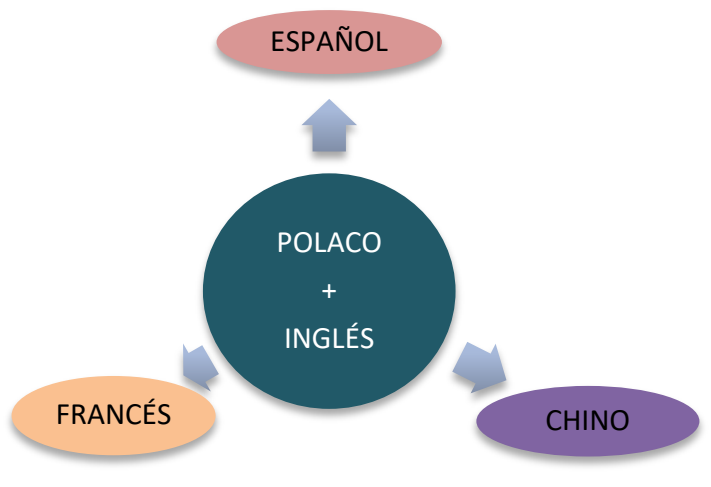

Figura 1. Idiomas en los que se imparte docencia en la escuela Internacional Trilinge de Varsovia.

En los primeros años de enseñanza infantil (hasta los 6 años de edad) la escolarización del alumnado se desarrolla según la proporción de $40 \%$ del programa escolar en inglés, $40 \%$ en el idioma elegido -en el caso a estudio, español-) y $20 \%$ en polaco. En la escuela primaria las proporciones cambian, dado que la escuela necesita seguir las reglas más estrictas del ministerio de educación nacional y entonces el $30 \%$ de todas las clases se impartan en inglés, $30 \%$ en polaco y $30 \%$ en español. Lo que diferencia estas proporciones son materias que se imparten en tres idiomas y materias que se enseñan solo en bilingüe (polaco e inglés).

En la escuela primaria dos son las materias, matemática y ciencias naturales, que se enseñan únicamente en bilingüe, es decir en: inglés + polaco. En trilingüe se enseña la literatura y las ciencias sociales (con historia y geografia), es decir en: ingles+ polaco + español / francés / chino. 


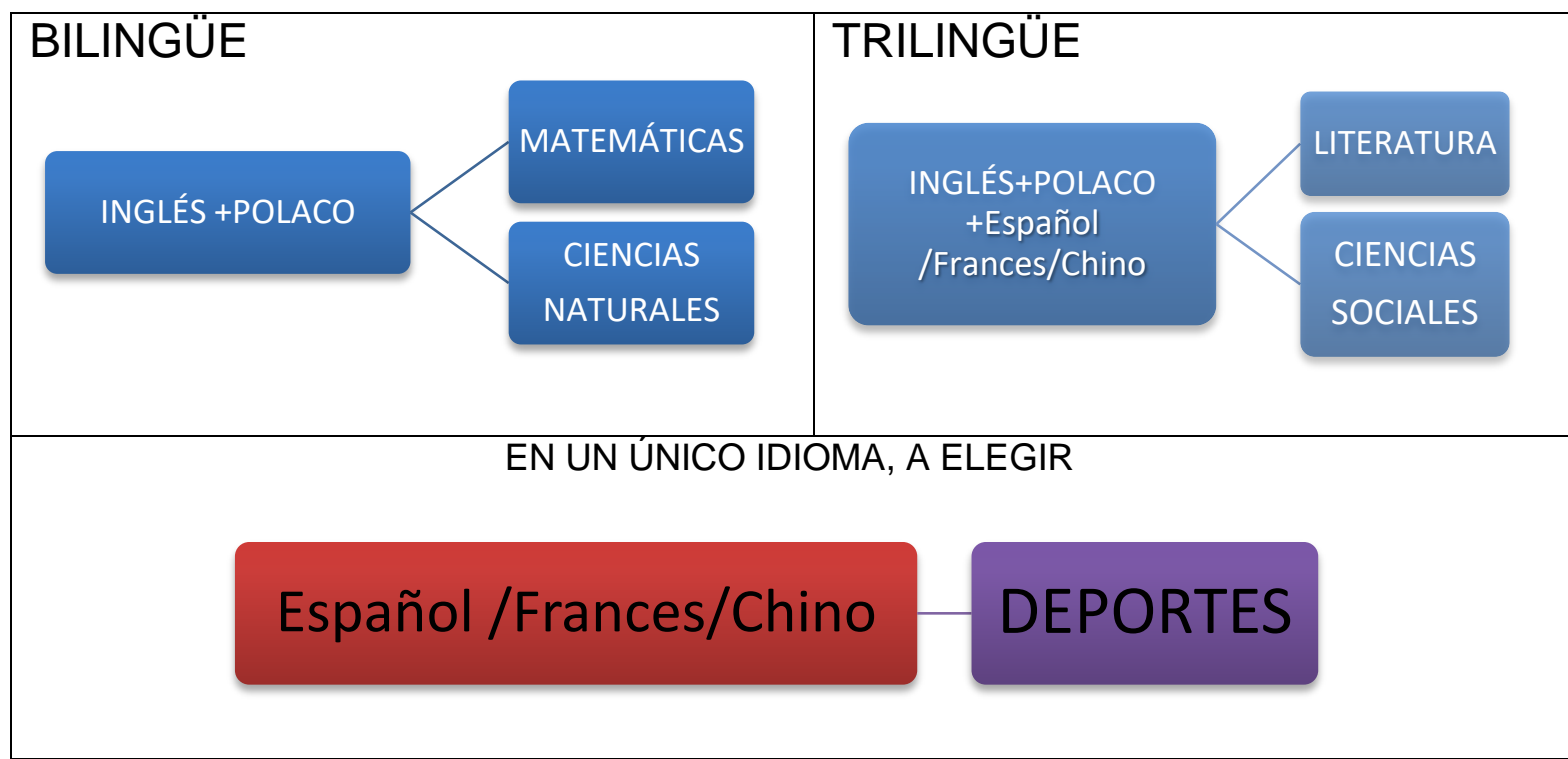

Figura 2. Idiomas en los que se imparten las diferentes materias en la escuela Internacional Trilinge de Varsovia.

Las actividades deportivas ocupan un lugar importante y se enseñan siempre en español, francés o chino, dependiendo de la selección realizada. La escuela enseña 4 horas de materias relacionadas con deporte $(\mathrm{EF})$ semanalmente. El alumnado de los grados 1 a 8 tiene cada semana una hora de baile, una hora de natación y dos horas de actividades deportivas.

Tabla 1

Distribución de las horas de actividad fisica (EF) por semana

\begin{tabular}{|c|c|c|}
\hline & \multicolumn{3}{c|}{ DEPORTE EN ESPANOL $(4 \mathrm{~h} / \mathrm{sem})$} \\
\hline BAILE & NATACIÓN & ACTIV. DEPORTIVAS \\
(1h) & $(\mathbf{1 h})$ & (2h) \\
\hline
\end{tabular}

Para las clases se considera que los y las alumnas hablan todos y todas español de una manera fluida, con un nivel mínimo de B2. No obstante, los niños y niñas que empiezan el aprendizaje de español a partir de la escuela primaria están también admitidos a las clases, pero reciben fuera de las clases de deporte, clases de refuerzo $(6$ horas semanales de clases individuales) para aprender el idioma y llegar al nivel B2 en un año.

\section{La Metodología}

La escuela internacional trilingüe de Varsovia utiliza una combinación de metodologías para abordar la enseñanza del español a través de las 
actividades deportivas que permiten mezclar niños y niñas de niveles lingüisticos varios.

La preparación del programa educativo y funcionamiento de las clases relacionadas con deporte están basadas en tres reglas principales:

a) El principio de AICLE

Permite combinar los estándares de diferentes currículos, es decir, el contenido de las clases es igual en las clases conducidas en polaco, en inglés y en español (ver Gómez Muñiz, 2013). Se elige la Educación Física y la enseñanza de las actividades deportivas como elemento vehicular puesto que esta materia, por sus características (se hace a base de muchos elementos corporales), ayuda a la comunicación general en el idioma español, también en el caso de los niños y niñas que no dominan el idioma o que empiezan a aprenderlo.

b) El principio de la inmersión lingüistica.

Las clases en cada materia están impartidas por el personal nativo en cada idioma cualificado para enseñar la materia en su país de origen.

c) El principio del método OPOL (una persona un idioma).

Durante las sesiones deportivas, el profesorado nunca se dirije al alumnado en otro idioma que no sea su idioma nativo, lo que permite identificar el idioma con el profesorado como representante del mismo. En las clases de deporte el contexto cultural es muy importante pues el concepto corporal de cada cultura es independiente y muestra la idiosincrasia de una sociedad (Dakowska, 2007).

Esto se refleja en todos los contenidos que se imparten en esta materia (natación, actividades deportivas y de forma evidente durante las sesiones de baile donde, además de los pasos y música proveniente de los países latinos, se enseña también la expresión corporal del baile, un modo interno de expresión, los vestidos tradicionales de las distintas regiones Latinoamericanas.

En la siguiente tabla (Tabla 2) se muestra a modo de ejemplo algunos de los contenidos que se imparten en esta materia.

Tabla 2

Ejemplo de contenidos que se desarrollan en español en las sesiones de deportes (Educación Fisica)

\begin{tabular}{|c|c|c|}
\hline & & CONTENIDOS \\
\hline \multirow{2}{*}{ 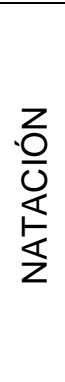 } & $\begin{array}{l}1^{\circ}, 2^{\circ} \mathrm{y} 3^{\circ} \\
\text { Ciclo de } \\
\text { primaria }\end{array}$ & $\begin{array}{l}\text { - Diagnóstico } \\
\text { - Flotación } \\
\text { - Natación básica } \\
\text { - Técnicas: crol y espalda } \\
\text { - Saltos, giros y equilibrios }\end{array}$ \\
\hline & $\begin{array}{l}4^{\circ}, 5^{\circ} \text { y } 6^{\circ} \\
\text { Ciclo de } \\
\text { primaria }\end{array}$ & $\begin{array}{ll}\text { - } & \text { Diagnóstico } \\
\text { - } & \text { Técnicas: Crol, espalda, braza y mariposa. } \\
\text { - } & \text { Entradas al agua, virajes y equilibrios }\end{array}$ \\
\hline
\end{tabular}




\begin{tabular}{|c|c|c|}
\hline \multirow{2}{*}{$\underset{\infty}{\stackrel{\varpi}{\rightleftarrows}}$} & $\begin{array}{l}1^{\circ}, 2^{\circ}{\mathrm{y} 3^{\circ}} \\
\text { Ciclo de } \\
\text { primaria }\end{array}$ & $\begin{array}{l}\text { - Conocimiento de las habilidades de los estudiantes en } \\
\text { el baile por medio de actividades que le permitan crear } \\
\text { y realizar movimientos corporales con ritmo. } \\
\text { - Conocimiento de bailes relacionados con la cultura de } \\
\text { España. } \\
\text { - Preparación por parte de los estudiantes para la } \\
\text { celebracion navideña. }\end{array}$ \\
\hline & $\begin{array}{l}4^{\circ}, 5^{\circ} \text { y } 6^{\circ} \\
\text { Ciclo de } \\
\text { primaria. }\end{array}$ & $\begin{array}{l}\text { - Coordinación de segmentos y coordinación óculo- } \\
\text { manual, agilidad en diferentes desplazamientos (lateral, } \\
\text { espaldas, saltos una pierna y piernas juntas). } \\
\text { - Equilibrio en diferentes desplazamientos, flexibilidad y } \\
\text { elasticidad. } \\
\text { - Conocimiento de bailes relacionados con la cultura de } \\
\text { España. }\end{array}$ \\
\hline \multirow[b]{2}{*}{ 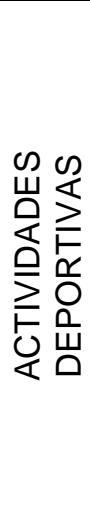 } & $\begin{array}{l}1^{\circ}, 2^{\circ} \text { y } 3^{\circ} \\
\text { Ciclo de } \\
\text { primaria }\end{array}$ & \multirow{2}{*}{ 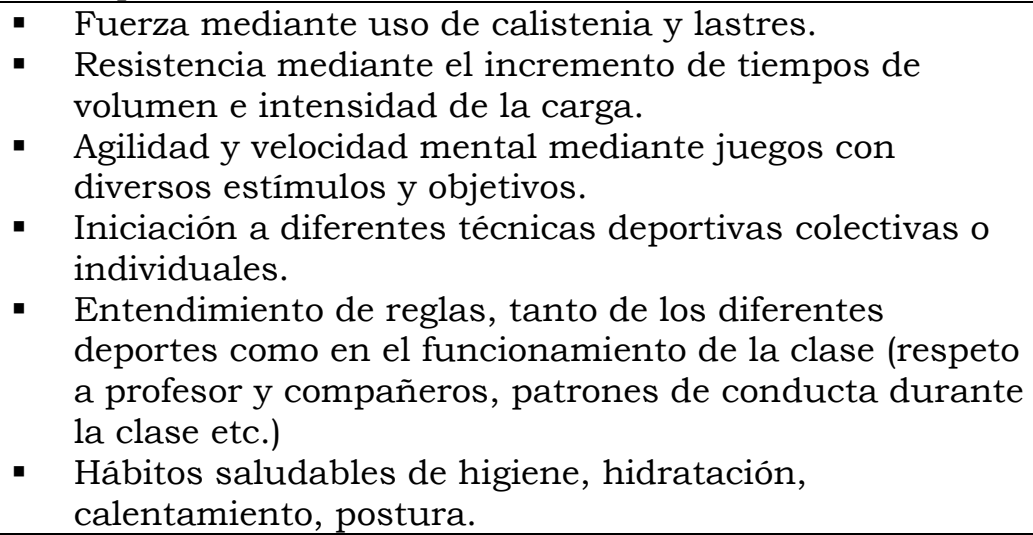 } \\
\hline & $\begin{array}{l}4^{\circ}, 5^{\circ} \text { y } 6^{\circ} \\
\text { Ciclo de } \\
\text { primaria }\end{array}$ & \\
\hline
\end{tabular}

\section{Análisis de datos}

Para tratar los datos obtenidos en el estudio se realizaron análisis descriptivos y correlacionales utilizando el paquete estadístico SPSS.

\section{Resultados}

Tras el análisis de los resultados se observa que, el 62,7\%del alumnado tiene como lengua materna el polaco, el $2 \%$ español, otro $2 \%$ son bilingües polaco - español y el resto tiene otros idiomas como lengua materna (Ver tabla 3).

Tabla 3

Porcentaje de alumnado en función de su lengua materna

\begin{tabular}{c|l|l|c|c|c|c} 
& $\begin{array}{l}\text { Pola } \\
\text { co }\end{array}$ & $\begin{array}{l}\text { Espa } \\
\text { nol }\end{array}$ & $\begin{array}{l}\text { Polaco y } \\
\text { Español }\end{array}$ & $\begin{array}{l}\text { Polaco y } \\
\text { otro }\end{array}$ & $\begin{array}{l}\text { Un idioma materno } \\
\text { no Polaco }\end{array}$ & $\begin{array}{l}\text { Dos diferentes al } \\
\text { Polaco }\end{array}$ \\
\hline$\%$ & 62,7 & 2 & 2 & 11,8 & 18,6 & 2,9
\end{tabular}


Si nos fijamos en el nivel que han adquirido en relación con el número de años que han estudiado español en la escuela de Varsovia, se observa que un $50 \%$ del alumnado necesita entre 3 y 5 años para adquirir el nivel $\mathrm{B} 1 \mathrm{y}$ un $25 \%$ para adquirir el nivel $\mathrm{C} 1$.

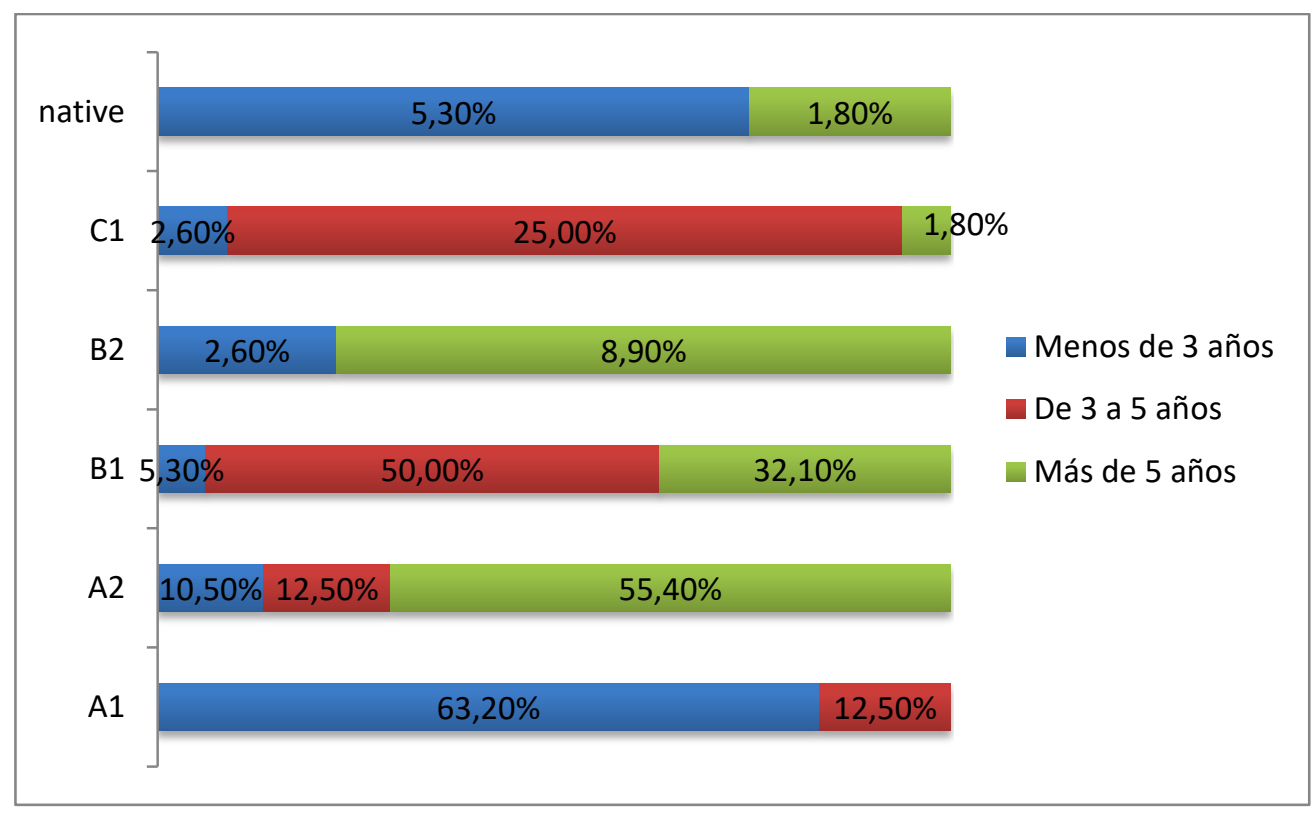

Figura 3. Años de español en la Escuela Trilingüe y nivel alcanzado.

Los datos de la nota media obtenida de las tres actividades deportivas, se estudiaron en comparación con la nota obtenida en español y el nivel que el alumnado tiene en este idioma. Se observa que el 100\% de las y los nativos españoles obtienen una nota de sobresaliente en idioma español, así como el $50 \%$ de quienes se encuentran en el nivel $\mathrm{C} 1$. Al analizar la nota en actividades deportivas (no se ha tenido en cuenta el hecho de ser nativo, por ser una muestra demasiado pequeña), el $100 \%$ de quienes tienen un nivel $\mathrm{C} 1$ han obtenido Notable, así como el $83,3 \%$ de quienes tienen el B2 y el 79,2\% de los que tienen el B1. 


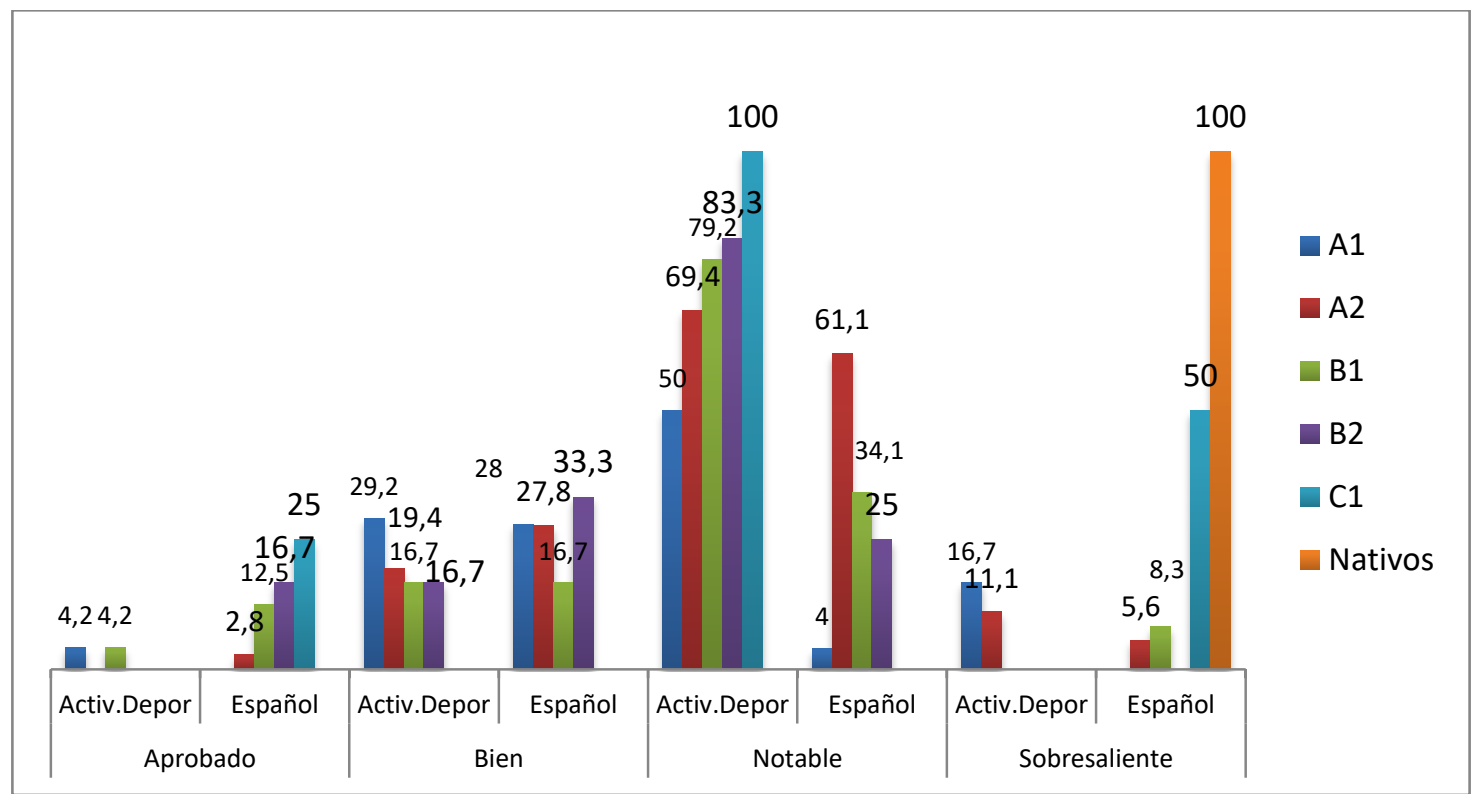

Figura 4. Porcentaje de alumnos y nota que obtienen en los contenidos del idioma español y las Actividades Deportivas.

De entre los alumnos/as que tienen nivel de español A1, el 50\% ha obtenido una nota de notable y el $16 \%$ de sobresaliente, y entre quienes tienen el nivel A2, el 69\% ha obtenido notable y el 11\% sobresaliente.

Se realizó una comparación de medias (ANOVA) para determinar la posible relación entre los años estudiando español y la nota obtenida en español y en actividades deportivas.

Tabla 3

Nota media obtenida en español y en actividades deportivas en función del tiempo que se lleva estudiando el idioma.

\begin{tabular}{cccccccccc}
\hline & \multicolumn{3}{c}{ Menos de 3 } & \multicolumn{2}{c}{ De 3 a 5 } & \multicolumn{2}{c}{ Más de 5 } \\
& & \multicolumn{2}{c}{ años } & años & \multicolumn{2}{c}{ años } & F(2,102) & $p$ \\
& $\mathrm{~N}$ & $\mathrm{M}$ & $\mathrm{DT}$ & $\mathrm{M}$ & $\mathrm{DT}$ & $\mathrm{M}$ & $\mathrm{DT}$ & & \\
\hline NotaEspañol & 102 & 2,11 & 2,31 & 4,25 & 1,98 & 4,63 & 0,96 & 26.306 & .000 \\
\hline NotasActDeport & 102 & 2,70 & 0,81 & 2,63 & 0,74 & 2,88 & 0,51 & 1.061 & .350 \\
\hline
\end{tabular}

Los datos indican una relación positiva y significativa entre los años que llevan los estudiantes aprendiendo español y la nota obtenida en dicha material $\mathrm{F}(2,99)=26,306, \mathrm{p}=.000$; sin embargo, no se observa relación entre los años estudiando español y la nota en actividades deportivas. 
Asimismo, se analizó la correlación entre la nota obtenida en español y la nota obtenida en el contenido "baile", por ser este un contenido relacionado con la cultura. Los análisis mostraron correlación positiva y significativa entre ambas variables.

Tabla 4

Relación entre la nota en la material español y baile

\begin{tabular}{|c|c|c|}
\hline & NotaEspañol & Nota_Baile \\
\hline NotaEspañol & 1 & $.203^{*}$ \\
\hline
\end{tabular}

\section{Discusión y conclusiones}

El objetivo de este estudio fue realizar un primer análisis sobre el posible efecto positivo del aprendizaje del español a través del modelo AICLE, en una experiencia que aborda el aprendizaje de dicho idioma, a través de actividades deportivas en una escuela trilingüe en Polonia.

La escuela internacional trilingüe de Varsobia, siguiendo las recomendaciones de la Comisión Europea (Resolucuón de la Comisión de la Unión Europea del 21 de noviembre de 2008), promueve la enseñanza de asignaturas no lingüísticas, como la educación física, en lenguas extranjeras a través del modelo AICLE. Así, en dicha escuela, los contenidos de educación física (natación, baile y actividades deportivas) son impartidos en español por profesorado nativo.

Los resultados muestran que el porcentaje de alumnado cuya lengua maternal es el español, es muy escaso, pero en todos los casos la nota obtenida por ellos/as tanto en actividades deportivas como en español, es de entre notable y sobresaliente. Sin embargo, en el conjunto del alumnado, no se aprecia relación entre la nota en actividades deportivas (EF) y español. Este hecho se podría explicar argumentando lo apuntado por Reloba, Chirosa, y Reigal (2016), quienes indican que a pesar de que la actividad física tiene una influencia positiva sobre los procesos cognitivos del alumnado, estos no parecen ser apreciables en su rendimiento académico a corto plazo.

En el presente estudios se ha probado una relación positiva y significativa entre los años de estudio de español y la nota obtenida en esa materia. En cuanto al nivel de español, la mitad del alumnado necesitó entre 3 y 5 años para adquirir un nivel B1, y en ese mismo periodo de tiempo, el 25\% llegó al nivel C1; es decir, en ese periodo de tiempo el $75 \%$ del alumnado obtiene un nivel igual o superior al B1.

También se observó que un alto porcentaje de quienes tienen un nivel de español bajo (A1 y A2), obtienen una nota alta en educación física (notable y sobresaliente). Este resultado se sitúa en la línea de los autores que defienden que los idiomas se utilizan como elementos de transmisión y adquisición de contenidos linguísticos y académicos en situaciones reales que giran alrededor de la comunicación y el significado (Cummins 2002; 
García, 2005; Ramos y Ruíz Omenaca, 2011). Asimismo, este resultado ayuda a argumentar los estudios que han demostrado que la motricidad y el desarrollo cognitivo mantienen una destacada relación (Barajas, 2013) y que además favorece el conocimiento del entorno y del propio protagonista.

Bolaños (1991) indica que los niños y niñas, a través del movimiento pueden lograr aprender a mejorar su rendimiento en todos los ámbitos de su vida. De ello se deduce que la práctica de actividades deportivas supone un entorno que favorece la adquisición de un alto nivel de español. Por otro lado, algunos lingüistas como Barron-Hauwaert (2004) recuerdan que los resultados que se obtienen cuando cada persona de referencia habla en su propia lengua, tienen efecto sobre el desarrollo cerebral del niño contribuyendo a que domine totalmente los idiomas a edades tempranas. En este caso, el hecho de que el profesorado que imparte las actividades deportivas sea nativo, podría ayudar en la adquisición del nivel más alto; sin embargo, sería necesario un estudio más profundo que reforzara estos argumentos.

Finalmente se observa una relación positiva y significatiba entre el contenido "baile" y la nota obtenida en español. McKay (2012) sostiene que la cultura influye en la enseñanza de idiomas de dos maneras: lingüistica y pedagógica. En este caso, esta relación se atribuye al contenido cultural de las herramientas de comunicación y la base cultural de la metodología de enseñanza, pues como indica Dakowska (2007), en las clases de deporte el contexto cultural es muy importante ya que el concepto corporal de cada cultura es independiente y muestra la idiosincrasia de una sociedad.

Para concluir se puede decir que el modelo utilizado en la escuela internacional trilingüe de Varsobia desarrollando la materia de educación fisica (actividades deportivas) con profesorado nativo, obtiene en un periodo de entre 3 y 5 años un alto nivel de español. No se ha podido establecer relación entre el aprendizaje de actividades deportivas y el nivel lingüístico, por ello se ve la necesidad de realizar estudios longitudinales que ayuden a verificar la posible influencia positiva de la actividad fisica sobre los procesos cognitivos del alumnado, pues coincidimos con Reloba, Chirosa, y Reigal (2016), en que estos no parecen ser apreciables en su rendimiento académico a corto plazo. Sin embargo, se ha podido establecer relación entre el contenido "baile" y la nota de español, por lo que se deduce que aquellos contenidos que por sus caracteristicas existe mayor proximidad entre cultura e idioma, favorecen el aprendizaje de este último. Asimismo, atendiendo a los resultados académicos de quienes tienen un nivel bajo de español, el contexto de las actividades deportivas se vislumbra como favorable en el desarrollo de la competencia motriz.

\section{Referencias}

Barajas, Y. (2013). Implementación de estrategias lúdicas para mejorar el 
desempeño escolar del área de matemáticas en los estudiantes de grado $6^{\circ} \mathrm{B}$ del colegio Gimnasio el Bosque de la ciudad de San José de Cúcuta. Revista actividad física y desarrollo humano. 119-126.

Barron-Hauwaert, S. (2004). Language strategies for bilingual families: The one-parent-one-language approach (No. 7). Multilingual Matters.

Bolaños, G. (1991). Educación por medio del movimiento y expresión corporal. San José, Costa Rica: Editorial EUNED.

Commission of the European Communities. (2003). Promoting language learning and linguistic diversity: An action plan 2004-2006.

Coyle, D. (2002). Relevance of CLIL to the European Commission Language Learning Objectives. In D. Marsh (Ed.), CLIL/EMILE European Dimension: Actions, Trends and Foresight Potential European Commission, Public Services Contract DG 3406/001-001.

Coyle, D., Hood, P., y Marsh, D. (2010). Content and language integrated learning. Ernst Klett Sprachen.

Cummins, J. (2002). Lenguaje, Poder y Pedagogía: Niños y Niñas Bilingües. Entre Dos Fuegos. Madrid: Morata.

Dakowska, M. (1995). Models of language use and language learning in the theory of language didactics. Rozprawy Uniwersytetu Warszawskiego.

Dakowska (2007). Models of Language Learning and Language Use in the Theory of Language Didactics. Warsaw University Editions

Dakowska, M. (2011). Teaching English as a foreign language: A guide for professionals. Wydawnictwo Naukowe PWN.

Garca, E. (2005). Teaching and Learning in Two Languages. NY: Teachers College Press.

Garrido, R., Giménez, M., Gil, P., Luciañez, A., Rodríguez, M. E., Romero, B., Rubio, M., y Sánchez, J. M. (2010). Experiencia con la competencia matemática en la clase de educación física. Revista de Didácticas Específicas. (2), 83-99.

Genesee, F. (1999). Learning through Two Languages: Studies of Immersion and Bilingual Education. Cambridge, MA: Newbury House.

Gómez Muñoz, S. (2013). La teoría de las inteligencias múltiples aplicada a la enseñanza con metodología AICLE. Universidad de Oviedo.

Grisolia, J. (2016). Interdisciplinariedad. Revista del Instituto de Estudios Interdisciplinarios en Derecho Social y Relaciones del Trabajo 
(IDEIDES). Rescatado

http://revista-

ideides.com/interdisciplinariedad/

Journal of the European Union Council Resultion of 21 November 2008 o

$\mathrm{n}$ a European Strategy for multilingualism (http://eur-

lex.europa.eu/LexUriServ/Lez.do?uri=OJ:C:2008:320:0001:0003:E $\mathrm{N}: \mathrm{P}: \mathrm{DF})$

Marsh, D. (1994). Bilingual education \& content and language integrated learning. International Association for Cross-cultural Communication (Eds.), Language Teaching in the Member States of the European Union (Lingua). Paris: University of Sorbonne.

McKay (2003). The Cultural Basis of Teaching English as an International Language. Chilean Context. Oxford University Press.

McKay, S. L. (2012). Principles of Teaching English as an International Language: Sandra Lee McKay. In Principles and practices for teaching English as an international language 36-54. Routledge.

Ramos, F., y Ruíz Omenaca, J. V. (2011). La educación física en centros bilingües de primaria inglés-español: de las singularidades propias del área a la elaboración de propuestas didácticas prácticas con AIBLE. Revista española de lingüística aplicada, 24, 153-170.

Reloba, S., Chirosa, L. J., y Reigal, R. E. (2016). Relación entre actividad física, procesos cognitivos y rendimiento académico de escolares: revisión de la literatura actual. Revista andaluza de medicina del deporte, 9(4), 166-172.

Rodríguez, M. y Vega, J. (2004). Relación entre la edad, el nivel de práctica de actividad física y el funcionamiento cognitivo. Tesis Licenciatura en Ciencias del Deporte con énfasis en Salud. Escuela Ciencias del Deporte de la Universidad Nacional. Heredia, Costa Rica.

Vollmer, Helmut Johannes (2006b). "Language Across the Curriculum Ways Towards Plurilingualism“. In: Martinyuk, Waldemar (ed.), Towards a Common European Framework of Reference for Language(s) of School Education. (Paper, presented at a conference in Kraków, 27-29 April 2006. Kraków: Jagellionian University. (to appear).

Woodburn, S. (1985). Desarrollo perceptual motor. Universidad Nacional. Heredia, Costa Rica: EUNA. 